\title{
Real-world treatment patterns and opioid use in chronic low back pain patients initiating duloxetine versus standard of care
}

This article was published in the following Dove Press journal:

Journal of Pain Research

25 November 2013

Number of times this article has been viewed

\author{
Jeffrey Scott Andrews' \\ Ning $\mathrm{Wu}^{2}$ \\ Shih-Yin Chen ${ }^{2}$ \\ $\mathrm{Xia} \mathrm{Yu^{2 }}$ \\ Xiaomei Peng' \\ Diego Novick' \\ 'Global Health Outcomes, Eli Lilly \\ and Company, Indianapolis, IN, USA; \\ ${ }^{2}$ Evidera, Lexington, MA, USA
}

Correspondence: Jeffrey Scott Andrews Lilly Corporate Center,

Indianapolis, IN 46285, USA

$\mathrm{Tel}+\mathrm{I} 3174526813$

Email andrews_jeffrey_scott@lilly.com
Abstract: To describe the use of pain medications in patients with chronic low back pain (CLBP) after initiating duloxetine or standard of care (SOC [muscle relaxants, gabapentin, pregabalin, venlafaxine, and tricyclic antidepressants]) for pain management, pharmacy and medical claims from Surveillance Data, Inc (SDI) Health were analyzed. Adult patients with CLBP who initiated duloxetine or SOC between November 2010 and April 2011 were identified. Treatment initiation was defined as no pill coverage for duloxetine or SOC in the previous 90 days. Included patients had no opioid use in the 90 days before initiation. Propensity score matching was used to select patients with similar baseline demographic and clinical characteristics for duloxetine and SOC cohorts. Compliance with index medication was assessed via medication possession ratio (MPR) and proportion of days covered (PDC) for 6 months after initiation. The proportion of patients receiving opioids and days on opioids after index date were assessed, and regression models were estimated to compare opioid use between cohorts. A total of 766 patients initiated duloxetine and 6,206 patients initiated SOC. After matching, 743 patients were selected for the duloxetine (mean age 57 years; female 74\%) and SOC (mean age 57 years; female 75\%) cohorts, respectively. Of the duloxetine cohort, $92 \%$ started on or below recommended daily dose $(\leq 60 \mathrm{mg})$. The duloxetine cohort had significantly higher MPR ( 0.78 versus [vs] 0.60$)$ and PDC ( 0.50 vs 0.31 ), were less likely to use opioids ( $45 \%$ vs $61 \%$ ), and had fewer days on opioids (median 0 vs 7 days) than the SOC cohort (all $P<0.001$ ). After adjusting for demographic and clinical characteristics, the duloxetine cohort initiated opioids later than the SOC cohort (hazard ratio $0.77,95 \%$ confidence interval 0.66-0.89). CLBP patients initiating duloxetine had better compliance with initiated medication and were less likely to use opioids than those initiating SOC.

Keywords: medications, duloxetine, low back pain, opioid

\section{Introduction and background}

Chronic low back pain (CLBP) is defined as low back pain (LBP) lasting longer than 3 months or occurring episodically within a 6-month time period. ${ }^{1}$ LBP affects a reported $5.6 \%$ of US adults each day, with lifetime prevalence estimated to be at least $60 \%-70 \% .^{2-4}$ The burden associated with treating back pain is significant, representing the third most common reason for surgical procedures and fifth most common reason for hospitalization. ${ }^{5}$ Americans spend at least \$US100 billion on LBP each year. ${ }^{67}$ CLBP accounts for $75 \%-90 \%$ of these costs. ${ }^{8}$

The treatment goal of CLBP is to reduce pain, maintain function, and prevent future exacerbation. ${ }^{9} 10$ Guidelines from the American College of Physicians and the American Pain Society recommend the use of medications with proven benefits in 
conjunction with patient education, self-care, and behavioral therapy. ${ }^{1,11-13}$ A variety of non-pharmacological interventions are recommended for patients who do not improve with self-care, including spinal manipulation, exercise therapy, massage, acupuncture, yoga, cognitive-behavioral therapy, progressive relaxation, and intensive interdisciplinary rehabilitation. ${ }^{10}$ Invasive procedures can be considered in the case of significant nerve root impingement or spinal stenosis and are recommended for those who fail to receive adequate relief from non-surgical treatments. ${ }^{1,11-14}$

In addition, a wide range of medications are suggested for the management of CLBP. ${ }^{11}$ However, evidence on the effectiveness and risks associated with their long-term use is limited. There is a need for additional long-term, real-world evidence to further describe the treatment patterns and effectiveness measures, such as treatment compliance, associated with medications commonly used in the management of CLBP. Common pharmacologic agents recognized in treatment guidelines for the management of CLBP include acetaminophen, non-steroidal anti-inflammatory drugs (NSAIDs), muscle relaxants, corticosteroids, and opioid analgesics. Additionally, antidepressants and anticonvulsants are also prescribed for the management of CLBP. ${ }^{1,11,13}$ Although acetaminophen and NSAIDs are considered first-line medications for CLBP, the benefits and risks associated with long-term use in the management of chronic pain are not well studied. ${ }^{11,15}$ Long-term use at high doses may increase the risk of experiencing adverse events, such as liver toxicity, renal and gastrointestinal damage, and cardiovascular events. ${ }^{16}$ Muscle relaxants are usually added to the treatment regimen when CLBP cannot be adequately controlled via the first-line treatments. However, clinical trials have shown that muscle relaxants are not more effective in reducing pain than NSAIDs. ${ }^{17}$ Evidence derived from clinical trials has been inconsistent on the efficacy of benzodiazepines, anticonvulsant drugs, and antidepressants on pain or functional outcomes among patients with LBP, and no clinically significant benefit has been found in clinical trials assessing systemic corticosteroids on the management of LBP. ${ }^{18}$

Another growing problem in the management of CLBP is the increasing use of opioids despite a lack of clinical evidence demonstrating the effectiveness of long-term opioid use in the management of chronic non-cancer pain. ${ }^{19-21}$ Increased use of opioids has been associated with higher levels of health care costs, emergency room visits, and death due to opioid overdose. ${ }^{19,22-24}$ A national survey revealed that the opioid prescriptions for spinal disorders increased by $108 \%$ between 1997 and 2004. ${ }^{19,22}$ There is a need for medications that can effectively manage pain while minimizing the use of opioids in individuals with chronic pain.

Duloxetine was approved in November 2010 by the US Food and Drug Administration for the management of chronic musculoskeletal pain, including CLBP. ${ }^{25}$ Unlike other common medications prescribed for CLBP, duloxetine works by inhibiting the uptake of 5-hydroxytryptamine (serotonin) and norepinephrine, which in turn results in the attenuation of persistent and chronic pain. ${ }^{26}$ Compared with placebo-treated patients, duloxetine-treated patients reported significantly less pain and maintained improvement in other health outcomes, such as physical function and quality of life, with a safety profile similar to that found in previous duloxetine trials. ${ }^{27-29}$ However, no studies have assessed the long-term effectiveness of duloxetine in CLBP patients in real-world settings.

The objectives of this retrospective database study were to characterize patients with CLBP who initiated duloxetine or standard of care (SOC), describe real-world treatment patterns, and compare subsequent opioid utilization among matched cohorts.

\section{Methods}

\section{Data source and sample selection}

In this study, the Surveillance Data, Inc (SDI) database from November 2009 to October 2011 was analyzed. SDI, part of IMS Health, maintains a data warehouse that contains billing records and claims of health care services, goods, and procedures provided in all associated health care providers including hospitals, private practitioners, and pharmacies. Data components in the SDI were abstracted directly from billing forms submitted by health care providers to SDI, such as CMS-1500 Private Practitioner Medical Claims completed for physician office visits, National Council for Prescription Drug Programs prescription claims submitted for prescriptions dispensed via retail pharmacy, and Hospital Charge Description Master for generating a patient's bill in hospital settings. SDI also maintains a separate file that documents the volume of claims or billing records submitted by each provider over time, which can be used to assess whether providers consistently submit data to IMS.

Patients with CLBP who initiated duloxetine or another second-line treatment prescribed for the management of CLBP between November 2010 and April 2011 were selected. The SOC group included muscle relaxants, gabapentin, pregabalin, venlafaxine, and tricyclic antidepressants (TCAs), and was based on common pain medications prescribed for the management of CLBP in real-world practice. ${ }^{18}$ 
Acetaminophen and NSAIDs were not included in the SOC because they are recognized in treatment guidelines as firstline treatments and many of them are available over the counter and are not captured in administrative health claims records. Treatment initiation was defined as no index medication coverage in the prior 90 days, and the date of treatment initiation was set as the index date. CLBP was defined as patients with at least two claims with associated diagnosis suggesting LBP (International Classification of Diseases, Ninth Revision, Clinical Modification [ICD-9-CM] ${ }^{30}$ codes 722.10 , $722.83,722.93,724.02,724.2 x, 724.3 x, 724.5 x, 724.8 x$ ) that were at least 90 days apart during the 12 months prior to the index date. Multiple claims were required over this defined time frame to effectively reflect the chronic nature associated with the LBP. Patients were excluded if they were aged less than 18 years on the index date, had received an opioid prescription within 90 days prior to the index date, or had medical claims for other indications of duloxetine and the SOC during the 6 months prior to the index date, where other indications include major depressive disorder, generalized anxiety disorder, epilepsy, diabetic peripheral neuropathic pain (DPNP), and post-herpetic neuralgia (PHN). Patients receiving care from providers that did not consistently submit data to SDI in the 6 months before and the 6 months after the index date were also excluded. Unlike typical administrative claims databases from insurance plans, which track enrollees' medical encounters across settings, SDI data consist of claims or billing records submitted by health care providers. This exclusion criterion requiring consistent data submission from providers minimizes the likelihood of selecting patients with incomplete records of medical encounters during the 12-month study period. Selected patients were assigned to duloxetine or SOC cohorts based on the medication initiated on the index date. Patients who initiated multiple medications were excluded.

Propensity score (PS) matching was used to select patients with similar baseline demographic and clinical characteristics from eligible duloxetine and SOC initiators. PS was the probability that patients initiated duloxetine during the study period; it was estimated from a logistic regression model with whether patients initiated duloxetine as the outcome. Potential predictors of initiating duloxetine were selected based on literature review, clinical reasoning, and descriptive analysis of pre-matched sample, and entered into the model in groups. The model with the best performance included age, gender, geographic region of residence, primary payment source of the patient, indicators of comorbidities, indicators of pain medication use before the index date, and whether patients had emergency room visits, outpatient hospital visits, or durable medical equipment use before the index date. PS was estimated as the predicted probability of initiating duloxetine given the patient's values for predictors. To assess whether the PS matching minimized the imbalance of patients' baseline characteristics between cohorts, demographics, comorbidities, and medication use in the 6-month period before the index date were summarized and compared

Table I Pre- and post-matching demographics and comorbidities 6 months before index date

\begin{tabular}{|c|c|c|c|c|}
\hline & \multicolumn{2}{|l|}{ Pre-match } & \multicolumn{2}{|l|}{ Post-match } \\
\hline & Duloxetine & soc & Duloxetine & SOC \\
\hline Number of patients & 766 & 6,206 & 743 & 743 \\
\hline Age (mean, SD) & $\begin{array}{l}57.2 \\
(14.9)\end{array}$ & $\begin{array}{l}58.9 \\
(15.4)^{*}\end{array}$ & $\begin{array}{l}57.3 \\
(14.9)\end{array}$ & $\begin{array}{l}57.1 \\
(14.7)\end{array}$ \\
\hline \multicolumn{5}{|l|}{ Age categories (\%) } \\
\hline $18-44$ & 19.7 & 18.4 & 19.5 & 19.8 \\
\hline $45-64$ & 48.2 & 44.3 & 48.2 & 48.6 \\
\hline $65-74$ & 17.9 & 19 & 17.9 & 18.4 \\
\hline $75-79$ & 6.9 & 8.3 & 7 & 6.1 \\
\hline $80+$ & 7.3 & 10 & 7.4 & 7.1 \\
\hline Female (\%) & 74.2 & $66.9 *$ & 74.0 & 74.7 \\
\hline Region (\%) & & $* *$ & & \\
\hline Northeast & 28.5 & 24.0 & 27.9 & 26.5 \\
\hline Midwest & 16.1 & 16.8 & 16.3 & 15.1 \\
\hline South & 36.7 & 39.8 & 36.7 & 40.5 \\
\hline West & 18.8 & 19.4 & 19.1 & 17.9 \\
\hline Insurance type (\%) & & $* *$ & & \\
\hline Commercial & 52.5 & 52.4 & 53.3 & 53.3 \\
\hline Medicaid & 10.2 & 9.2 & 10.1 & 9.7 \\
\hline Medicare & 30.4 & 29.1 & 29.7 & 29.7 \\
\hline Other/unknown & 6.9 & 9.3 & 6.9 & 7.3 \\
\hline \multicolumn{5}{|l|}{ Comorbidities (\%) } \\
\hline Hypertension & 28.6 & $36.7^{*}$ & 28.5 & 27.6 \\
\hline Diabetes & 17.5 & 16.0 & 17.6 & 17.9 \\
\hline $\begin{array}{l}\text { Chronic } \\
\text { respiratory disease }\end{array}$ & 12.1 & 11.3 & 12.2 & 13.2 \\
\hline Depression & 6.7 & $2.2 *$ & 6.1 & 5.7 \\
\hline $\begin{array}{l}\text { Cerebrovascular } \\
\text { disease }\end{array}$ & 4.4 & 4.0 & 4.4 & 4.3 \\
\hline Skin ulcers/cellulitis & 3.8 & 3.4 & 3.6 & 3.9 \\
\hline Neuropathic pain & 4.0 & $2.5^{*}$ & 4.0 & 3.5 \\
\hline Any malignancy & 3.4 & 4.7 & 3.4 & 3.6 \\
\hline $\begin{array}{l}\text { Peripheral vascular } \\
\text { disease }\end{array}$ & 3.3 & 3.0 & 3.4 & 3.0 \\
\hline $\begin{array}{l}\text { Congestive heart } \\
\text { failure }\end{array}$ & 2.1 & 2.2 & 2.2 & 1.5 \\
\hline Renal disease & 2.0 & 3.1 & 1.9 & I.I \\
\hline Peptic ulcer & 0.9 & 0.8 & 0.8 & 1.2 \\
\hline $\begin{array}{l}\text { Rheumatologic } \\
\text { disease }\end{array}$ & 0.9 & 0.7 & 0.9 & 0.8 \\
\hline $\begin{array}{l}\text { Myocardial } \\
\text { infarction }\end{array}$ & 0.4 & 0.8 & 0.4 & 0.3 \\
\hline
\end{tabular}

Notes: *Statistically significant at $P<0.05$ (reference: duloxetine). **Distribution of region and insurance type were significantly different between two cohorts. SOC included gabapentin, pregabalin, venlafaxine, muscle relaxants, or tricyclic antidepressants.

Abbreviations: SD, standard deviation; SOC, standard of care. 
between the duloxetine and SOC cohorts for unmatched and matched cohorts, respectively (Table 1).

Patient baseline characteristics, including demographic characteristics, comorbid conditions, and medication use in the 6 months prior to the index date were summarized for all patients who met the inclusion/exclusion criteria and for matched control patients. The initial daily dose of duloxetine was assessed for the duloxetine cohort and compared with the recommended dose for CLBP (ie, $60 \mathrm{mg}$ per day), and the proportion of patients starting above, below, or on the recommended dose was estimated. The proportion of patients who adjusted the daily dose during the 6-month follow-up period was also estimated.

Patient adherence to the index medication was assessed using several methods. Medication possession ratio (MPR) was calculated as the days' supply of medication dispensed during the specified follow-up period divided by the number of days between the first and last prescription refill. Proportion of days covered (PDC) was calculated as total days supplied over a 6-month follow-up period, divided by 183 . Discontinuation of therapy was defined as a gap of $60+$ days in treatment. The date of therapy discontinuation was defined as the last day with access to the index medication before the 60-day treatment gap. Opioid use in the 6 months following the index date was also examined by assessing the proportion of patients using opioids, days on opioid, morphine-equivalent dosage, and time to opioid initiation. In addition, separate analyses were conducted for short-acting and long-acting opioids. Short-acting opioids are characterized as having a quick onset of action and short duration of analgesic activity and included immediate-release codeine, fentanyl, hydrocodone, hydromorphone, meperidine, morphine, oxycodone, oxymorphone, pentazocine, propoxyphene, and tramadol. ${ }^{31}$ Long-acting opioids included methadone, and levorphanol and extended-release or controlled-release forms of opioids previously listed. Opioids not targeting pain (eg, codeine for coughing) were removed from the analysis.

\section{Statistical analysis}

Study outcomes assessed after the index date were only reported for the matched sample. To detect statistically significant differences between cohorts, the chi-square test was used for categorical variables, the Student's $t$-test for continuous variables, and the non-parametric Wilcoxon test for count variables. Kaplan-Meier curves were plotted and Cox proportional hazard models were estimated to compare the relative risk of opioid initiation and the relative risk of discontinuation of the index medication between cohorts. All analyses were conducted using SAS 9.2 (SAS Institute, Inc, Cary, NC, USA). Differences were considered statistically significant if $P<0.05$.

\section{Results}

\section{Sample selection}

Of the 76,991 patients with CLBP identified from the SDI data, 25,338 initiated duloxetine or SOC between November 2010 and April 2011. After applying the selection criteria, 766 duloxetine initiators and 6,206 SOC initiators (muscle relaxants $61.8 \%$, gabapentin $23.4 \%$, pregabalin $5.4 \%$, venlafaxine $2.5 \%$, and TCAs $6.9 \%$ ) were identified based on inclusion/ exclusion criteria. After PS matching, 743 patients were selected from each cohort (Figure 1).

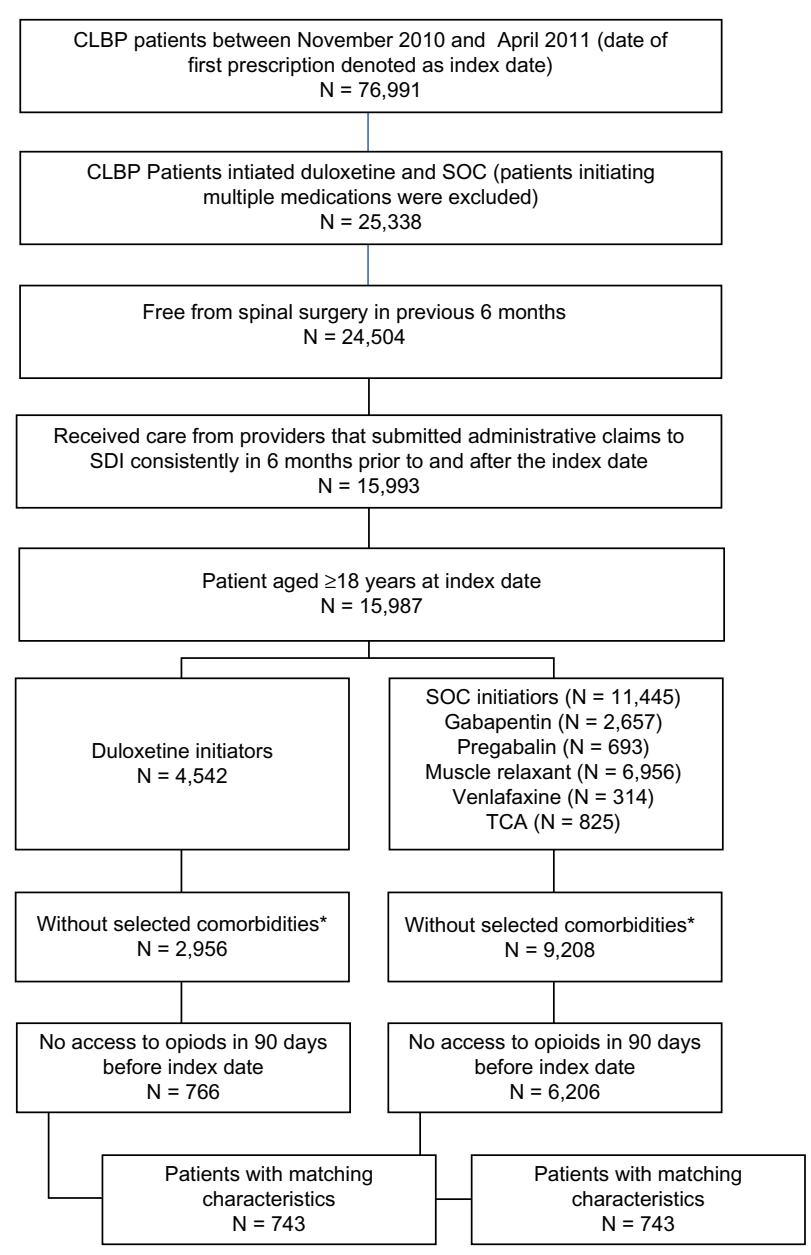

Figure I Sample selection flowchart.

Notes: *Patients with the following comorbidities were excluded: major depressive disorder, generalized anxiety disorder, epilepsy, diabetic peripheral neuropathic pain, and post-herpetic neuralgia.

Abbreviations: CLBP, chronic low back pain; SDI, Surveillance Data, Inc; SOC, standard of care; TCA tricyclic antidepressant. 


\section{Baseline characteristics}

Before matching, the duloxetine cohort was younger (duloxetine 57.2 years; SOC 58.9 years; $P<0.05$ ) and had a higher proportion of females $(74.2 \%$ versus [vs] $66.9 \%$; $P<0.05$ ) than the SOC cohort (Table 1). A majority of both cohorts were enrolled in commercial insurance plans, and about one-third in Medicare. The duloxetine cohort had a significantly lower proportion of patients with hypertension ( $28.6 \%$ vs $36.7 \%)$, but a higher proportion with depression $(6.7 \%$ vs $2.2 \%)$ and neuropathic pain ( $4.0 \%$ vs $2.5 \%)$ than the SOC cohort (all $P<0.05$ ).

In the PS matched sample, we did not observe significant differences in demographics or prevalence of comorbidities between the matched cohorts. The matched cohorts had an average age of 57 years. A similar proportion were females (duloxetine $74.0 \%$; SOC $74.7 \% ; P=0.77$ ), and the prevalence of comorbidities was not significantly different between cohorts.

Table 2 Pre- and post-matching medication utilization

\begin{tabular}{|c|c|c|c|c|}
\hline & \multicolumn{2}{|l|}{ Pre-match } & \multicolumn{2}{|l|}{ Post-match } \\
\hline & Duloxetine & soc & Duloxetine & SOC \\
\hline \multicolumn{5}{|c|}{ Pre-index medication utilization } \\
\hline Benzodiazepines (\%) & 28.5 & $16.7^{*}$ & 27.1 & 27.1 \\
\hline Muscle relaxants (\%) & 20.8 & $16.2 *$ & 19.9 & 20.6 \\
\hline Antidepressants (\%) & 40.2 & $13.3^{*}$ & 38.6 & 37.7 \\
\hline Duloxetine & 13.1 & $3.2^{*}$ & 12.4 & 12.9 \\
\hline $\begin{array}{l}\text { Selective serotonin } \\
\text { reuptake inhibitors }\end{array}$ & 16.7 & $6.0^{*}$ & 15.5 & 15.2 \\
\hline $\begin{array}{l}\text { Tricyclic } \\
\text { antidepressants }\end{array}$ & 5.4 & $1.9 *$ & 5.2 & 4.2 \\
\hline Pregabalin (\%) & 7.0 & $1.5^{*}$ & 5.8 & 5.9 \\
\hline Gabapentin (\%) & 14.0 & $4.6^{*}$ & 12.8 & 10.6 \\
\hline $\begin{array}{l}\text { Non-Steroidal Anti- } \\
\text { inflammatory drugs (\%) }\end{array}$ & 29.8 & $22.2 *$ & 29.5 & 31.6 \\
\hline Celecoxib & 5.7 & $3.9 *$ & 5.8 & 5.2 \\
\hline Oral steroids (\%) & 16.2 & 16.2 & 15.9 & 17.2 \\
\hline Opioids (\%) & 26.6 & $27.4^{*}$ & 25.7 & 26.6 \\
\hline Long-acting opioids & 2.3 & $1.3^{*}$ & 2.3 & 1.7 \\
\hline Short-acting opioids & 25.8 & 27.0 & 24.9 & 25.8 \\
\hline Number of unique & 7.8 & 6.4 & 7.6 & 7.6 \\
\hline medications: mean (SD) & $(5.3)$ & $(4.5)^{*}$ & $(5.2)$ & $(5.3)$ \\
\hline \multicolumn{5}{|c|}{ Dose of index medication on index date $(\%)^{\S}$} \\
\hline Above recommended & 8.8 & - & 8.5 & - \\
\hline Below recommended & 42.7 & - & 48.7 & - \\
\hline Recommended & 48.6 & - & 42.8 & - \\
\hline \multicolumn{5}{|c|}{ Change of dose of index medication during 6 months after index date (\%) } \\
\hline Decrease & 6.5 & - & 6.7 & - \\
\hline Increase & 13.2 & - & 13.5 & - \\
\hline No change & 49.5 & - & 48.5 & - \\
\hline One refill & 30.8 & - & 31.4 & - \\
\hline
\end{tabular}

Notes: *Statistically significant at $P<0.05$ (reference: duloxetine); 'stest was not performed between cohorts. SOC included gabapentin, pregabalin, venlafaxine, muscle relaxants, or tricyclic antidepressants.

Abbreviations: SD, standard deviation; SOC, standard of care.
Of all eligible patients with CLBP, the duloxetine cohort was more likely than the SOC cohort to use medications commonly prescribed for CLBP in the pre-index period, with the exception of oral steroids and opioids (Table 2). The SOC cohort was significantly more likely to use opioids (27.4\% vs $26.6 \% ; P<0.05)$. The most commonly used medications associated with the treatment of CLBP were antidepressants (duloxetine cohort 40.2\%; SOC cohort 13.3\%), NSAIDs (duloxetine cohort 29.8\%; SOC cohort 22.2\%), benzodiazepines (duloxetine cohort 28.5\%; SOC cohort 16.7\%), and opioids (duloxetine cohort 26.6\%; SOC cohort $27.4 \%$ ) (all $P<0.05$ ). On average, the duloxetine cohort used significantly more unique medications during the 6 months prior to the index date ( 7.8 vs $6.4 ; P<0.05$ ) than the SOC cohort. In the matched sample, pre-index medication use was similar between the duloxetine and SOC cohorts $(P>0.05$ for all post-match baseline medication use); over one-third in both the duloxetine and the SOC cohorts used antidepressants, with selective serotonin reuptake inhibitors more frequently used than duloxetine or TCAs, about one-third in both cohorts used NSAIDs, and over one-quarter in both cohorts used benzodiazepines. On average, patients in each cohort received 7.6 medications in the pre-index period.

\section{Use of index medication}

The initiating dose and dose titration of duloxetine during the 6-month follow-up was also assessed (Table 2). Of all eligible duloxetine initiators (pre-match), $42.7 \%$ started duloxetine on the recommended dose ( $60 \mathrm{mg}$ daily), and $48.6 \%$ below the recommended dose. A majority of the patients in the duloxetine cohort either remained on the initiating dose $(49.5 \%)$ or did not have a refill $(30.8 \%)$ during the 6-month post-index follow-up period. Additionally, $13.2 \%$ had a dose increase, while $6.5 \%$ had a dose decrease. In the matched sample, the proportion starting at the recommended dose was slightly lower (pre-match $48.6 \%$; post-match $42.8 \%$ ) than the unmatched sample, and the pattern of dose titration was similar to that observed in the unmatched sample.

Compared with the SOC cohort, the duloxetine cohort, on average, had higher MPR (mean [standard deviation; SD] 0.78 [0.25] vs 0.60 [0.32]; $P<0.05)$ and a higher proportion with MPR $\geq 0.8(60.8 \%$ vs $35.8 \% ; P<0.05)$ (Table 3$)$. The duloxetine cohort also had higher PDC (mean [SD] 0.50 [0.31] vs $0.31[0.28] ; P<0.05$ ) and a higher proportion with PDC $\geq 0.8$ ( $25.6 \%$ vs $10.5 \% ; P<0.05)$. The duloxetine cohort was less likely to discontinue the index medication in the 6 months after the index date $(56.7 \%$ vs $79.7 \% ; P<0.05)$, and time to discontinuation of the index medication was later than for the 
Table 3 Compliance with index medication among matched cohorts

\begin{tabular}{lll}
\hline & Duloxetine & SOC \\
\hline Compliance with index medication & & \\
Mean MPR (SD) & $0.78(0.25)$ & $0.60(0.32)^{*}$ \\
MPR $\geq 0.8(\%)$ & 60.8 & $35.8^{*}$ \\
Mean PDC (SD) & $0.50(0.3 \mathrm{I})$ & $0.3 \mathrm{I}(0.28)^{*}$ \\
PDC $\geq 0.8(\%)$ & 25.6 & $10.5^{*}$ \\
Number of days on medication & $92.9(56.0)$ & $54.8(49.8)$ \\
Discontinuation (\%) & 56.7 & $79.7^{*}$ \\
\hline
\end{tabular}

Notes: *Statistically significant at $P<0.05$ (reference: duloxetine). SOC included gabapentin, pregabalin, venlafaxine, muscle relaxants, or tricyclic antidepressants.

Abbreviations: MPR, medication possession ratio; PDC, proportion of days covered; SD, standard deviation; SOC, standard of care.
SOC cohort (median 99 vs 30 days; $P<0.05$ ) (Figure 2A). After controlling for baseline demographic and clinical characteristics, the duloxetine cohort discontinued the index medication significantly later than SOC cohort (hazard ratio $0.49,95 \%$ confidence interval $[\mathrm{CI}] 0.43-0.55$ ) (Figure $2 \mathrm{~A}$ ).

\section{Opioid use after the initiation} of index medication

Compared with the SOC cohort, duloxetine patients were less likely to use opioids (45.1\% vs $60.7 \%)$, reflected in lower utilization of both short-acting (44.3\% vs $59.9 \%)$ and
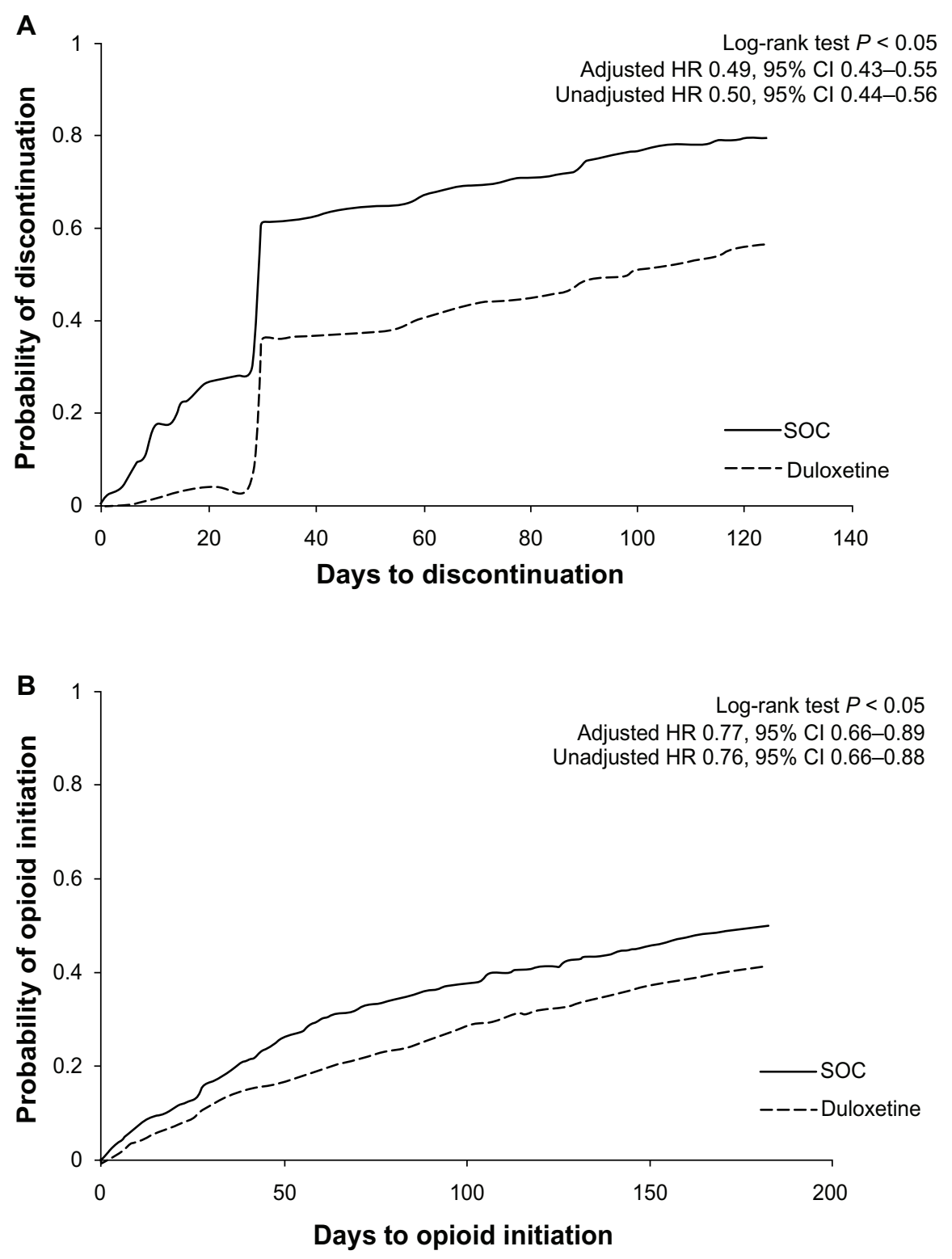

Figure 2 Time to index medication discontinuation and first opioid use in 6-month post-index period among matched cohorts.

Notes: (A) Time to index medication discontinuation in 6-month post-index period among matched cohorts. (B) Time to first opioid use in 6-month post-index period among matched cohorts. Adjusted HR controlled for baseline demographic and clinical characteristics. SOC included gabapentin, pregabalin, venlafaxine, muscle relaxants, or tricyclic antidepressants.

Abbreviations: $\mathrm{Cl}$, confidence interval; HR, hazard ratio; SOC, standard of care. 
Table 4 Opioid use in the 6-month post-index period among matched cohorts

\begin{tabular}{lll}
\hline & Duloxetine & SOC \\
\hline Opioid use (\%) & 45.1 & $60.7^{*}$ \\
$\quad$ Long-acting opioids & 4.2 & $7.0^{*}$ \\
$\quad$ Short-acting opioids & 44.3 & $59.9^{*}$ \\
$\begin{array}{l}\text { Days on opioid, median } \\
\text { (25th, 75th percentile) }\end{array}$ & $0(0,19)$ & $7(0,30)^{*}$ \\
$\begin{array}{l}\text { Morphine equivalent dosage in milligrams, } \\
\text { median (25th, 75th percentile) }\end{array}$ & $0(0,510)$ & $198(0,900)^{*}$ \\
\hline
\end{tabular}

Notes: *Statistically significant at $P<0.05$ (reference: duloxetine). SOC included gabapentin, pregabalin, venlafaxine, muscle relaxants, or tricyclic antidepressants. Abbreviation: SOC, standard of care.

long-acting opioids ( $4.2 \%$ vs $7.0 \%)$ (all $P<0.05)$ (Table 4$)$. On average, the duloxetine cohort had fewer days' supply of opioids (median 0 vs 7 days; $P<0.05$ ), and received less morphine equivalent dosage (median 0 vs $198 \mathrm{mg} ; P<0.05$ ) during the post-6-month index period. Compared with the
SOC cohort, the time to first initiating an opioid in the postindex period was later for the duloxetine cohort (duloxetine cohort: 25 th percentile $=86$ days, median $>183$ days [median cannot be estimated because $<50 \%$ of patients used opioids during the 183-day follow-up]; SOC cohort: 25th percentile $=47$ days, median $=181$ days; $P<0.05$ ) (Figure 2B). After controlling for baseline demographic and clinical characteristics, the duloxetine cohort initiated opioids significantly later than did the SOC cohort (hazard ratio: $0.77,95 \%$ CI $0.66-0.89$ ) (Table 5).

\section{Discussion}

Findings from this study suggested that patients with CLBP who initiated duloxetine treatment had presumably greater compliance with the treatment and had less opioid exposure than patients initiating other pain medications commonly prescribed for CLBP. To our knowledge, no previous work

Table 5 Relative risk of index medication discontinuation or opioid initiation: duloxetine versus standard of care

\begin{tabular}{|c|c|c|c|c|}
\hline \multirow[t]{2}{*}{ Variable } & \multicolumn{2}{|c|}{ Relative risk of index treatment discontinuation } & \multicolumn{2}{|c|}{ Relative risk of opioid initiation } \\
\hline & HR & $95 \% \mathrm{Cl}$ & HR & $95 \% \mathrm{Cl}$ \\
\hline Duloxetine & 0.49 & $(0.43-0.55)$ & 0.77 & $(0.66-0.89)$ \\
\hline \multicolumn{5}{|l|}{ Age (years) } \\
\hline $18-44$ & Reference & & Reference & \\
\hline $45-64$ & 0.95 & $(0.80-1.12)$ & 0.79 & $(0.65-0.96)$ \\
\hline $65-74$ & 0.84 & $(0.67-1.05)$ & 0.79 & $(0.60-1.04)$ \\
\hline $75-79$ & 0.92 & $(0.67-1.25)$ & 0.75 & $(0.51-1.10)$ \\
\hline $80+$ & 0.93 & $(0.69-1.25)$ & 0.81 & $(0.56-1.16)$ \\
\hline \multicolumn{5}{|l|}{ Gender } \\
\hline Female & Reference & & Reference & \\
\hline Male & 1.03 & $(0.90-1.19)$ & 1.03 & $(0.87-1.23)$ \\
\hline \multicolumn{5}{|l|}{ Region } \\
\hline West & Reference & & Reference & \\
\hline Midwest & 0.77 & $(0.63-0.94)$ & $\mathrm{I} .44$ & $(1.13-1.84)$ \\
\hline Northeast & 0.91 & $(0.78-1.06)$ & 1.40 & $(1.15-1.70)$ \\
\hline South & 0.87 & $(0.72-1.04)$ & 1.19 & $(0.94-1.52)$ \\
\hline \multicolumn{5}{|c|}{ Primary source of payment } \\
\hline Commercial & Reference & & Reference & \\
\hline Medicare & 0.90 & $(0.76-1.08)$ & $\mathrm{I} .14$ & $(0.91-1.42)$ \\
\hline Medicaid & 0.84 & $(0.67-1.05)$ & 1.14 & $(0.88-1.47)$ \\
\hline Unknown & 0.90 & $(0.69-1.16)$ & 0.93 & $(0.69-1.27)$ \\
\hline \multicolumn{5}{|c|}{ Charlson Comorbidity Index } \\
\hline 0 & Reference & & Reference & \\
\hline 1 & 0.88 & $(0.75-1.05)$ & 1.06 & $(0.87-1.29)$ \\
\hline 2 & 0.99 & $(0.8 \mathrm{I}-1.20)$ & 1.02 & $(0.81-1.29)$ \\
\hline 3 & 1.09 & $(0.88-1.36)$ & 0.91 & $(0.69-1.20)$ \\
\hline $4+$ & 1.02 & $(0.8 \mathrm{I}-1.28)$ & 1.09 & $(0.83-1.44)$ \\
\hline \multicolumn{5}{|c|}{ Drug use in pre-index period } \\
\hline Acetaminophens & 1.06 & $(0.72-1.55)$ & 0.74 & $(0.45-1.22)$ \\
\hline Anticonvulsants & 0.75 & $(0.63-0.90)$ & 0.92 & $(0.75-1.13)$ \\
\hline Antidepressants & 0.82 & $(0.72-0.94)$ & 1.00 & $(0.85-1.17)$ \\
\hline Opioids & 1.04 & $(0.90-1.20)$ & 1.85 & $(1.58-2.18)$ \\
\hline Muscle relaxants & 0.76 & $(0.65-0.91)$ & 1.08 & $(0.89-1.30)$ \\
\hline NSAIDs & 1.12 & $(0.97-1.28)$ & 1.09 & $(0.93-1.29)$ \\
\hline Steroids & 0.96 & $(0.81-1.13)$ & 1.11 & $(0.91-1.34)$ \\
\hline Benzodiazepines & 0.90 & $(0.78-1.04)$ & 1.14 & $(0.96-1.36)$ \\
\hline
\end{tabular}

Notes: Hazard ratio <I: discontinue index medication or initiate opioid later than the reference group; hazard ratio > I: discontinue index medication or initiate opioid sooner than the reference group. Standard of care included gabapentin, pregabalin, venlafaxine, muscle relaxants, or tricyclic antidepressants.

Abbreviations: $\mathrm{Cl}$, confidence interval; HR, hazard ratio; NSAIDs, non-steroidal anti-inflammatory drugs. 
has explored treatment patterns, compliance, and opioid utilization following the approval of duloxetine for CLBP. The results of the study may help address the current lack of long-term, real-world evidence associated with the use of commonly prescribed pain medications in the treatment of CLBP that is noted in treatment guidelines and evidence reviews.

One of the primary treatment goals for CLBP is to alleviate pain. Adherence to pain medications prescribed for CLBP is essential to achieve and maintain adequate pain relief. In a recent survey, primary care physicians in community clinics were asked to identify barriers to achieving optimal pain control for patients with chronic pain. A majority of physicians cited psychological factors including depression and anxiety (76.6\%) and poor compliance with recommendations $(53.1 \%)$ as the main reasons. ${ }^{32}$ In the current study, the proportion of patients with MPR or PDC equal to or greater than $80 \%$ was almost twice as high in patients who initiated duloxetine than in patients who initiated other second-line treatments. This finding may have important implications for achieving treatment outcomes in CLBP, and additional research is warranted to explore the drivers of better treatment compliance associated with duloxetine. In particular, potential differences regarding efficacy and tolerability could be impacting on treatment compliance and warrant further investigation.

The reduction in post-index opioid utilization observed in the duloxetine cohort of this study is consistent with findings from previous duloxetine research. Less opioid use may be associated with better compliance with the initiated treatment in the duloxetine cohort. Such a mechanism was suggested by other studies on duloxetine use among patients with chronic pain conditions. ${ }^{33,34}$ Zhao et al found that, among patients with DPNP who initiated duloxetine or SOC for DPNP, persistent duloxetine users had a greater reduction in opioid use after duloxetine initiation than non-persistent users and those who initiated SOC for DPNP. ${ }^{34}$ Additional research is needed to explore the relationship between treatment selection, compliance, and reduction in opioid use associated with the treatment of chronic pain conditions.

The unique mechanism of action of duloxetine, via selective serotonin and norepinephrine reuptake inhibition, may provide a further explanation for the observed differences in treatment outcomes. CLBP is considered a mixed-pain syndrome, with the potential for multifactorial etiology and pain mechanisms, including nociceptive, neuropathic, and hyperalgesic components. ${ }^{35,36}$ Duloxetine is believed to act upon descending inhibitory pain pathways, ${ }^{37}$ and has demonstrated efficacy in a variety of other chronic pain conditions, including DPNP, ${ }^{38,39}$ fibromyalgia, ${ }^{40,41}$ and pain associated with osteoarthritis of the knee. ${ }^{42}$ Future studies, such as long-term pragmatic clinical trials or patient survey, physician survey, or chart review that collect data on effectiveness of treatment and reasons for treatment discontinuation or switch, are needed to confirm the drivers of treatment outcomes in CLBP, including better compliance.

There has been a pressing need for treatment alternatives for the management of CLBP due to limitations associated with existing options. Guidelines published by the American College of Physicians and the American Pain Society do not recommend long-term use of opioids, especially the long-term use of short-acting opioids, for the management of non-cancer chronic pain. The short-acting opioids are recommended for opioid initiation and reserved for breakthrough pain. There has been limited evidence demonstrating the benefits of long-term use of opioids for the treatment of CLBP. ${ }^{17,18}$ A systematic literature review conducted in 2007 only identified one clinical trial demonstrating that the addition of opioids to a naproxen regimen provided greater pain relief than naproxen alone. ${ }^{43}$ In addition, side effects are common in opioid users and often lead to treatment discontinuation. This limits opioids as a potential routine treatment for the management of chronic pain. The recent increase in opioid use has been associated with more emergency room visits, deaths due to opioid overdose, and greater likelihood for opioid abuse and addiction resulting in greater negative clinical consequences and higher overall health care costs. ${ }^{19,22,44}$ Despite the limitations and lack of evidence associated with opioid use in the management of CLBP, a rapid increase in use persists. ${ }^{45,46}$

Non-opioid pharmacological agents used in the treatment of CLBP are also associated with a variety of limiting adverse events. Traditional NSAIDs are associated with gastrointestinal events and liver and renal toxicities. Long-term use of NSAIDs poses high risks for CLBP patients. In the current study, CLBP patients were on average 57 years old. It is common to observe decreased renal or liver function and multiple comorbidities in this population, potentially exposing patients to a greater risk of developing drug overdoses, drug-drug interactions, and adverse events. ${ }^{47}$ Newer NSAIDs, cyclooxygenase-2 selective inhibitors, are associated with a lower risk of gastrointestinal events than traditional NSAIDs, but carry an increased risk of myocardial infarction, stroke, and thrombosis. ${ }^{48,49}$ Antidepressants, such as TCAs, have shown mild to moderate effects on the management of LBP 
compared with placebo. ${ }^{50}$ Duloxetine has been shown to be efficacious in managing CLBP in clinical trials, and adverse events reported in these trials have been mild and transitory in nature. ${ }^{27-29,51}$

This study had several limitations. The SDI data were compiled from providers. If patients received care from providers other than those included in the SDI network, the health care encounters or prescriptions dispensed cannot be captured in the SDI data. All medical conditions were identified based on ICD-9-CM diagnosis codes recorded in an administrative claims database. The diagnoses cannot be confirmed via medical charts or laboratory results, and the severity of pain cannot be assessed. Although the database shows what medications were prescribed to patients, diagnoses were not recorded for prescribed medications. Thus, the exact condition for which a medication was prescribed cannot be determined. Rate of inpatient hospitalization is under-documented in the SDI data. Due to the incomplete information on resource utilization, we were unable to assess association between treatment compliance and economic outcomes. However, most of the care for CLBP is provided in outpatient settings and the incomplete inpatient records should have a minimal impact on the presented analysis. Provider prescription habits regarding opioids may have represented an additional confounder. However, given the limited information about the providers in the SDI data, we were unable to assess the association between provider prescription habits and study outcomes. Furthermore, a small number of initiators were identified for some of the study medications in the SOC cohort, making it difficult to conduct comparisons between individual medications and duloxetine. For example, 338 patients initiated pregabalin, 154 initiated venlafaxine, and 426 initiated TCAs in the pre-matching SOC cohort. After matching, the sample size of these sub-cohorts became too small to conduct individual comparisons. Finally, although the PS matching improved the comparability of baseline clinical and demographic characteristics between duloxetine and SOC cohorts, unobservable confounders might have biased the estimates. As a result, the findings from this analysis can only be interpreted as association and not causation.

\section{Conclusion}

This study evaluated pain medication utilization in patients with CLBP after initiating duloxetine or SOC. Patients with CLBP initiating duloxetine had better compliance and a lower likelihood of opioid utilization than those initiating the SOC. The findings from this study could be informative to health care providers in managing CLBP. Future research may be warranted in order to assess the full economic implications of duloxetine use in the treatment of chronic pain.

\section{Acknowledgment}

The authors thank Alan Wang, Evidera, Lexington, MA, USA, for his contributions to this study and the preparation of this manuscript. Additionally, the authors thank Eli Lilly and Company for providing funding support.

\section{Disclosure}

The work reported here was funded by and performed for Eli Lilly and Company by Evidera. Authors XP, JSA, and DN are employees of Eli Lilly and Company. Authors NW, SYC, and XY are employed by Evidera, which provides consulting and other research services to pharmaceutical, device, government, and non-government organizations. In their salaried positions, they work with a variety of companies and organizations and are precluded from receiving payment or honoraria directly from these organizations for services rendered. The authors declare no other conflicts of interest in this work.

\section{References}

1. Krismer M, van Tulder M. Low Back Pain Group of the B, Joint Health Strategies for Europe Project. Strategies for prevention and management of musculoskeletal conditions. Low back pain (non-specific). Best Pract Res Clin Rheumatol. 2007;21(1):77-91.

2. Loney PL, Stratford PW. The prevalence of low back pain in adults: a methodological review of the literature. Phys Ther. 1999;79(4): 384-396.

3. Hart LG, Deyo RA, Cherkin DC. Physician office visits for low back pain. Frequency, clinical evaluation, and treatment patterns from a US national survey. Spine (Phila PA 1976). 1995;20(1):11-19.

4. van Tulder M, Koes B, Bombardier C. Low back pain. Best Pract Res Clin Rheumatol. 2002;16(5):761-775.

5. US Department of Health and Human Services. Healthy People 2020. Arthritis, osteoporosis, and chronic back conditions [webpage on the internet]. Available from: http://healthypeople.gov/2020/ topicsobjectives2020/overview.aspx?topicid=3. Accessed June 15, 2013.

6. Katz JN. Lumbar disc disorders and low-back pain: socioeconomic factors and consequences. J Bone Joint Surg Am. 2006;88 Suppl 2: 21-24.

7. Dagenais S, Caro J, Haldeman S. A systematic review of low back pain cost of illness studies in the United States and internationally. Spine $J$. 2008;8(1):8-20.

8. Kuijer W, Brouwer S, Preuper HR, Groothoff JW, Geertzen JH, Dijkstra PU. Work status and chronic low back pain: exploring the International Classification of Functioning, Disability and Health. Disabil Rehabil. 2006;28(6):379-388.

9. Wilson JF. In the clinic. Low back pain. Ann Int Med. 2008;148(9): ITC5-1-ITC5-16.

10. Miller SM. Low back pain: pharmacologic management. Prim Care. 2012;39(3):499-510. 
11. Chou R, Qaseem A, Snow V, et al. Diagnosis and treatment of low back pain: a joint clinical practice guideline from the American College of Physicians and the American Pain Society. Ann Int Med. 2007;147(7): 478-491.

12. Hestbaek L, Leboeuf-Yde C, Manniche C. Low back pain: what is the long-term course? A review of studies of general patient populations. Eur Spine J. 2003;12(2):149-165.

13. Goertz M, Thorson D, Bonsell J, Bonte B, Campbell R, Haake B, Johnson K, Kramer C, Mueller B, Peterson S, Setterlund L, Timming R. Adult acute and subacute low back pain. Institute for Clinical Systems Improvement (ICSI); 2012 Nov. Available from: https://www.icsi. org/_asset/bjvqrj/LBP.pdf. Accessed June 15, 2013.

14. Pengel LH, Herbert RD, Maher CG, Refshauge KM. Acute low back pain: systematic review of its prognosis. BMJ. 2003;327(7410):323.

15. Last AR, Hulbert K. Chronic low back pain: evaluation and management. Am Fam Physician. 2009;79(12):1067-1074.

16. Nourjah P, Ahmad SR, Karwoski C, Willy M. Estimates of acetaminophen (Paracetomal)-associated overdoses in the United States. Pharmacoepidemiol Drug Saf. 2006;15(6):398-405.

17. van Tulder MW, Touray T, Furlan AD, Solway S, Bouter LM. Muscle relaxants for non-specific low back pain. Cochrane Database Syst Rev. 2003;(2):CD004252.

18. Chou R, Huffman LH. American Pain Society; American College of Physicians. Medications for acute and chronic low back pain: a review of the evidence for an American Pain Society/American College of Physicians clinical practice guideline. Ann Int Med. 2007;147(7):505-514.

19. Compton WM, Volkow ND. Major increases in opioid analgesic abuse in the United States: concerns and strategies. Drug Alcohol depend. 2006;81(2):103-107.

20. Lee TJ. Pharmacologic treatment for low back pain: one component of pain care. Phys Med Rehabil Clin N Am. 2010;21(4):793-800.

21. Deyo RA, Mirza SK, Turner JA, Martin BI. Overtreating chronic back pain: time to back off? J Am Board Fam Med. 2009;22(1):62-68.

22. Paulozzi LJ, Budnitz DS, Xi Y. Increasing deaths from opioid analgesics in the United States. Pharmacoepidemiol Drug Saf. 2006;15(9): 618-627.

23. Mitchell JM. Utilization trends for advanced imaging procedures: evidence from individuals with private insurance coverage in California. Med Care. 2008;46(5):460-466

24. Martin BI, Deyo RA, Mirza SK, et al. Expenditures and health status among adults with back and neck problems. JAMA. 2008;299(6): 656-664.

25. Cymbalta ${ }^{\circledR}$ (duloxetine delayed-release capsules) [package insert]. Indianapolis, IN: Lilly USA, LLC; 2010 [webpage on the internet] Available from: http://pi.lilly.com/us/cymbalta-pi.pdf. Accessed July 24, 2012.

26. Iyengar S, Webster AA, Hemrick-Luecke SK, Xu JY, Simmons RM. Efficacy of duloxetine, a potent and balanced serotonin-norepinephrine reuptake inhibitor in persistent pain models in rats. $J$ Pharmacol Exp Ther. 2004;311(2):576-584.

27. Skljarevski V, Zhang S, Desaiah D, et al. Duloxetine versus placebo in patients with chronic low back pain: a 12-week, fixed-dose, randomized, double-blind trial. J Pain. 2010;11(12):1282-1290.

28. Skljarevski V, Zhang S, Chappell AS, Walker DJ, Murray I, Backonja M. Maintenance of effect of duloxetine in patients with chronic low back pain: a 41-week uncontrolled, dose-blinded study. Pain Med. 2010;11(5):648-657.

29. Skljarevski V, Ossanna M, Liu-Seifert H, et al. A double-blind, randomized trial of duloxetine versus placebo in the management of chronic low back pain. Eur J Neurol. 2009;16(9):1041-1048.

30. National Center for Health Statistics. Classification of Diseases and Injuries. Center for Disease Control. Available from: ftp://ftp.cdc.gov/ pub/Health_Statistics/NCHS/Publications/ICD-9/ucod.txt. Accessed April 18, 2012.
31. Argoff CE, Silvershein DI. A comparison of long- and short-acting opioids for the treatment of chronic noncancer pain: tailoring therapy to meet patient needs. Mayo Clin Proc. 2009;84(7):602-612.

32. Upshur CC, Luckmann RS, Savageau JA. Primary care provider concerns about management of chronic pain in community clinic populations. J Gen Intern Med. 2006;21(6):652-655.

33. Chen S, Wu N, Fraser K, Boulanger L, Zhao Y. Opioid use and healthcare costs among patients with DPNP initiating duloxetine versus other treatments. Curr Med Res Opin. 2010;26(10):2507-2516.

34. Zhao Y, Wu N, Chen S, Boulanger L, Police RL, Fraser K. Changes in opioid use and healthcare costs among US patients with diabetic peripheral neuropathic pain treated with duloxetine compared with other therapies. Curr Med Res Opin. 2010;26(9):2147-2156.

35. Freynhagen R, Baron R. The evaluation of neuropathic components in low back pain. Curr Pain Headache Rep. 2009;13(3):185-190.

36. Deyo RA, Weinstein JN. Low back pain. N Engl J Med. 2001;344(5): 363-370.

37. Robinson MJ, Edwards SE, Iyengar S, Bymaster F, Clark M, Katon W. Depression and pain. Front Biosci (Landmark Ed). 2009;14: 5031-5051.

38. Raskin J, Pritchett YL, Wang F, et al. A double-blind, randomized multicenter trial comparing duloxetine with placebo in the management of diabetic peripheral neuropathic pain. Pain Med. 2005;6(5):346-356.

39. Wernicke JF, Pritchett YL, D'Souza DN, et al. A randomized controlled trial of duloxetine in diabetic peripheral neuropathic pain. Neurology. 2006;67(8):1411-1420.

40. Arnold LM. Duloxetine and other antidepressants in the treatment of patients with fibromyalgia. Pain Med. 2007;8 Suppl 2:S63-S74.

41. Arnold LM, Pritchett YL, D'Souza DN, Kajdasz DK, Iyengar S, Wernicke JF. Duloxetine for the treatment of fibromyalgia in women: pooled results from two randomized, placebo-controlled clinical trials. J Womens Health (Larchmt). 2007;16(8):1145-1156.

42. Chappell AS, Ossanna MJ, Liu-Seifert H, et al. Duloxetine, a centrally acting analgesic, in the treatment of patients with osteoarthritis knee pain: a 13-week, randomized, placebo-controlled trial. Pain. 2009;146(3):253-260.

43. Lai KC, Chu KM, Hui WM, et al. Celecoxib compared with lansoprazole and naproxen to prevent gastrointestinal ulcer complications. Am J Med. 2005;118(11):1271-1278

44. Bohnert AS, Valenstein M, Bair MJ, et al. Association between opioid prescribing patterns and opioid overdose-related deaths. JAMA. 2011;305(13):1315-1321.

45. Boulanger $\mathrm{L}, \mathrm{Wu} \mathrm{N}$, Chen SY, et al. Predictors of pain medication selection among patients diagnosed with fibromyalgia. Pain Pract. 2012;12(4):266-275.

46. Kalso E, Edwards JE, Moore RA, McQuay HJ. Opioids in chronic non-cancer pain: systematic review of efficacy and safety. Pain. 2004;112(3):372-380.

47. Katz WA, Barkin RL. Dilemmas in chronic/persistent pain management. Dis Mon. 2010;56(4):233-250.

48. Antman EM, Bennett JS, Daugherty A, et al. Use of nonsteroidal antiinflammatory drugs: an update for clinicians: a scientific statement from the American Heart Association. Circulation. 2007;115(12): 1634-1642.

49. Caldwell B, Aldington S, Weatherall M, Shirtcliffe P, Beasley R. Risk of cardiovascular events and celecoxib: a systematic review and metaanalysis. J R Soc Med. 2006;99(3):132-140.

50. Staiger TO, Gaster B, Sullivan MD, Deyo RA. Systematic review of antidepressants in the treatment of chronic low back pain. Spine. 2003;28(22):2540-2545.

51. Brunton S, Wang F, Edwards SB, et al. Profile of adverse events with duloxetine treatment: a pooled analysis of placebo-controlled studies. Drug Saf. 2010;33(5):393-407. 
Journal of Pain Research

\section{Publish your work in this journal}

The Journal of Pain Research is an international, peer-reviewed, open access, online journal that welcomes laboratory and clinical findings in the fields of pain research and the prevention and management of pain. Original research, reviews, symposium reports, hypothesis formation and commentaries are all considered for publication.

The manuscript management system is completely online and includes a very quick and fair peer-review system, which is all easy to use. Visit http://www.dovepress.com/testimonials.php to read real quotes from published authors.

\footnotetext{
Submit your manuscript here: http://www.dovepress.com/journal-of-pain-research-journal
} 